\title{
Martin Orensanz*
}

\section{A Syntactic Approach to Meillassoux's Concept of Hyper-Chaos}

https://doi.org/10.1515/opphil-2020-0008

Received September 06, 2019; accepted January 02, 2020

Abstract: Axiomatic systems can be understood as subsets of syntactic systems. By a process of increasing abstraction, the notion of a syntactic system can become useful for understanding Meillassoux's concept of hyper-Chaos.

Keywords: Quentin Meillassoux; Rudolf Carnap; axiomatic system; syntactic system; hyper-Chaos

\section{Rudolf Carnap}

The impression that one gets when reading After Finitude is that Meillassoux's two main "philosophical heroes" are Descartes and Hume. However, to a lesser degree, he also offers some laudatory remarks on Karl Popper:

For we know - at least since Popper - that every theory advanced by empirical science is by right revisable: it can be falsified and supplanted by one that is more elegant, or that exhibits greater empirical accuracy. ${ }^{1}$

Elsewhere in his book, he criticizes some aspects of Popper's philosophy of science; namely, that it fails to provide an adequate solution to Hume's problem. However, his remark on what we have learned about scientific theories "at least since Popper" can mislead the reader into thinking that before Popper, philosophy of science does not have anything interesting to offer. On this point, just as Meillassoux "rescues" Popper and attempts to render his work relevant to readers of continental philosophy, we wish to rescue another figure, for the same reasons. We are referring to Rudolf Carnap.

It has become customary to regard Carnap as some sort of apolitical, ascetic individual, untroubled by economic, social and political problems. In the worst case scenario, he is sometimes portrayed as a narrowminded individual who spent his life in an ivory tower dealing with philosophically shallow topics such as inductive logic and the logical analysis of language.

As George Reisch ${ }^{2}$ has shown, that is not the case. Carnap was not apolitical nor a conservative, on the contrary, he was a left-winger. He was a militant socialist who believed that logic and science must contribute to the solution of economic, social and political problems. After he moved to the United States, he was persecuted during the Cold War, specifically during the era of McCarthyism. Even more so, he was investigated by the FBI under the suspicion of being a "soviet agent". Thanks to George Reich's work and insistence, the FBI has declassified their file on Carnap, which can now be downloaded from the Internet. Other members of the Vienna Circle, such as Otto Neurath, were also left-wingers who held

1 Meillassoux, After Finitude, 12.

2 Reisch, How the Cold War Transformed Philosophy of Science: To the Icy Slopes of Logic.

*Corresponding author: Martin Orensanz, National University of Mar del Plata, Mar del Plata, Argentina;

E-mail: martin7600@gmail.com 
similar views regarding logic and science. During the early 1960’s, Carnap published his autobiography, where he says:

I think that nearly all of us shared the following three views as a matter of course which hardly needed any discussion. The first is the view that man has no supernatural protectors or enemies and that therefore whatever can be done to improve life is the task of man himself. Second, we had the conviction that mankind is able to change the conditions of life in such a way that many of the sufferings of today may be avoided and that the external and the internal situation of life for the individual, the community, and finally for humanity will be essentially improved. The third is the view that all deliberate action presupposes knowledge of the world, that the scientific method is the best method of acquiring knowledge and that therefore science must be regarded as one of the most valuable instruments for the improvement of life. In Vienna we had no names for these views; if we look for a brief designation in American terminology for the combination of these three convictions, the best would seem to be "scientific humanism". ${ }^{3}$

He was not a narrow-minded individual either, nor are his works on logical syntax philosophically shallow. Carnap was a profound thinker, who fully grasped the high degree of abstraction, creativity and freedom that characterize the invention of new syntaxes. Even though what we are going to say may seem incredible to the reader, Deleuze \& Guattari agree with Carnap on this point. Although they emphasized the creation of philosophical concepts, they were also fully aware of the liberty, artistry and beauty that characterizes the creation of a syntax:

In each case there must be a strange necessity for these words and for their choice, like an element of style. The concept's baptism calls for a specifically philosophical taste that proceeds with violence or by insinuation and constitutes a philosophical language within language - not just a vocabulary but a syntax that attains the sublime or a great beauty. ${ }^{4}$

A syntax, as they say, is capable of attaining "the sublime or a great beauty". Carnap had similar ideas. For him, syntax was not merely "the boring part" of grammar or semiotics, and logical syntax was not some mindless, mechanical textbook exercise. On the contrary, he considered that mathematics and logic are branches of syntax. Huge branches indeed, but branches nonetheless. Or, to state it differently: if syntax was a book, then mathematics and logic would be two of its chapters, among others. Quite surely they would be the largest chapters in the book, spanning thousands or even millions of pages, but they would be chapters nonetheless. Thus, when Carnap speaks of "logical syntax", he has in mind any system of meaningless signs that can be chosen at will, and combined according to arbitrary, meaningless rules that are also chosen at will. In this sense, he has in mind something like the infinite variations of chess; any "alternative rules" for a variant of chess are equally legitimate from a formal point of view. Carnap condensed all of this in his famous Principle of Tolerance, which he presented in his Logical Syntax of Language. There he says:

Principle of Tolerance: It is not our business to set up prohibitions, but to arrive at conventions. Some of the prohibitions which have hitherto been suggested have been historically useful in that they have served to emphasize important differences and bring them to general notice. But such prohibitions can be replaced by a definitional differentiation. In many cases, this is brought about by the simultaneous investigation (analogous to that of Euclidean and non-Euclidean geometries) of language-forms of different kinds -for instance, a definite and an indefinite language, or a language admitting and one not admitting the Law of Excluded Middle. ${ }^{5}$

He was clearly aware of the profound consequences that non-Euclidean geometries had for philosophy. And it was also clear to him that, as Hilbert had argued, all syntaxes are equally legitimate from a purely formal point of view, just like all the alternative variants of chess are. This is why he says that one can invent "a language admitting and one not admitting the Law of Excluded Middle". Such a law is not an axiom in the Aristotelian ${ }^{6}$ sense of being a self-evident primary truth, but a syntactic convention instead. Just as one can

3 Carnap, "Intellectual Autobiography", 83.

4 Deleuze \& Guattari, What is Philosophy?, 8.

5 Carnap, Logical Syntax of Language, 51.

6 Aristotle, Aristotle’s Posterior Analytics, 6-20. 
develop a non-Euclidean geometry where Euclid's fifth postulate is negated, one can also invent a syntactic system in which the Law or Principle of Excluded Middle is negated. In fact, this applies to all four of the classical principles: the Principle of Identity, the Principle of Non-Contradiction, the Principle of Excluded Middle, and the Principle of Sufficient Reason. From Carnap's point of view, one can invent a language in which any, some or all of these principles are negated, without necessarily deducing any contradictions from such negations. For example, Meillassoux himself has opted for the negation of the Principle of Sufficient Reason. In his book, Carnap also presents his Principle of Tolerance in a more poetic way:

In logic, there are no morals. Everyone is at liberty to build up his own logic, i.e. his own form of language, as he wishes. All that is required of him is that, if he wishes to discuss it, he must state his methods clearly, and give syntactical rules instead of philosophical arguments. ${ }^{7}$

The preceding quote requires some comment, because it could lead to misinterpretations. Carnap is not against philosophical arguments per se. Rather, he is against the idea that one can dismiss a new logic, such as a non-classical logic, by means of philosophical arguments. Why? Because philosophical arguments already presuppose a syntax, and what is being discussed is a new syntax. Thus, if one invents a new logic, it would be inaccurate to dismiss it by way of a philosophical argument based on the syntax of a modus tollens, for example. When discussing a new logic, one has to examine the new syntactical rules of that system, instead of using the syntactic rules of other systems in order to dismiss it.

According to Meillassoux's definition of philosophy, there are "internal criteria for distinguishing between licit and illicit claims". ${ }^{8}$ What are these internal criteria, exactly? Meillassoux does not offer any specifications on this point. So we must elaborate it by ourselves. Initially, one option is that these "internal criteria" are what Klimovsky and Boido call "production rules" and what Carnap calls "syntactic rules", because these purely formal rules are what permit such a language to trace a distinction between well-formed formulas, on the one hand, and combinations of signs that are not well-formed formulas, on the other.

So, for example, the term being-in-the-world is a well-formed formula in the context of Heidegger's philosophy, more specifically in the context of Being and Time. Being-in-the-world, as a well-formed formula, is different from "being in the world", precisely because the former uses the "-" symbol between those words, while the latter does not. Heidegger thus created new syntactic rules that function as "internal criteria" for distinguishing between licit and illicit claims within the framework of his philosophy. Thus, the following quote is a well-formed formula:

Being-in-the-world is a state of Dasein which is necessary a priori, but it is far from sufficient for completely determining Dasein's Being. ${ }^{9}$

Since the preceding quote is a well-formed formula in the context of Heidegger's philosophy, it is also a licit claim in that context. On the other hand, an expression such as "Dasein-Being-State necessary determining world for" is not a well-formed formula, and thus it is an illicit claim. But such brutal examples are not the only ones that would be illicit claims in Heidegger's philosophy, because merely writing "being in the world" without the "-" sign between those words would also count as a formula which is not well-formed, and thus any claims that use such a formula would be illicit claims.

Both Badiou and Meillassoux rely heavily on axiomatic set theory. It seems to us that their philosophies could benefit by taking syntactic systems into account. The rationale for this claim is that such systems are more abstract and encompassing than axiomatic systems. The latter are subsets of the former. Here is how Klimovsky and Boido explain this point:

The notion of an axiomatic system, so markedly formal and abstract, has given way to an even more abstract notion, called syntactic system by some authors. Syntax, let us remember once again, refers to everything that involves signs and their combinations, while semantics contemplates the meaning and reference to entities exterior to the language. In fact,

7 Carnap, Logical Syntax of Language, 52.

8 Meillassoux, After Finitude, 76-77.

9 Heidegger, Being and Time, 79. 
a syntactic system has similarities with an axiomatic system, but its degree of abstraction and distance with respect to the meaning of the expressions and the semantic aspects of the language is far greater. What is a syntactic system? It is constituted by signs which have no meaning or category attributed to them, by arbitrary expressions or formulas constituted by finite successions of signs and, in particular, by a subclass of such expressions called well-formed expressions. The condition of "well-formed" of the expressions means here that they have been constructed according to a purely arbitrary criterion, but which indicates that those expressions must be used in a certain way and not another. ${ }^{10}$

It is important to highlight what they say about the degree of abstraction that characterizes syntactic systems. Mathematics in general and axiomatic systems in particular are already extremely abstract, but syntactic systems are even more abstract according to these authors. Syntax, understood in this way, is far from being simply "the boring part" of grammar or semiotics. Quite the contrary, it is one of the most abstract fields of inquiry, even more so than the whole of mathematics and logic. Formal axiomatic systems are a subset of syntactic systems. The difference between them concerns what Klimovsky and Boido call "the underlying logic". In a formal axiomatic system, the underlying logic must be a classical logic, meaning that it must be a deductive logic, such as propositional logic or predicate logic, for example. In a syntactic system, this is not a requirement. Instead of an underlying logic, what we have is a set of "production rules" or "transformation rules", which do not necessarily have to be deductive inferences:

The components of a syntactic system would be, thus, to begin: 1. signs; 2. expressions or formulas, that is, sequences of signs; 3. well-formed expressions according to certain rules of formation. Now we must choose some of these as the starting point of our manipulations, which are also called axioms, a peculiar combination of meaningless signs. Then, instead of logical rules, we have the transformation rules, or production rules, which allow us to obtain well-formed expressions from others. (Some authors call them rules of inference, but the word "inference" here has nothing to do with what has traditionally been called that, so it is preferable to avoid this nomenclature). And finally we have the theorems, which are the well-formed expressions obtained by the manipulations that the transformation rules allow from the starting-point expressions, the axioms. We emphasize that the transformation rules are completely conventional and arbitrary, and are not necessarily guided by grammatical or logical considerations. ${ }^{11}$

In other words, one can use a non-classical logic in a syntactic system. The example that these authors mention are the paraconsistent logics developed by the Brazilian logician Netwon da Costa. The Argentine logician F. G. Asenjo had also done work in non-classical logics. In these, one can negate a classical principle, such as the Principle of Non-Contradiction. In After Finitude, Meillassoux himself refers to Newton da Costa's work ${ }^{12}$ in order to refute a possible objection to his line of reasoning. But the price he pays to make that refutation work is rather high, since he traces a distinction between contradictory statements and contradictory entities, understood as real, non-linguistic entities. He says that the former exist while the latter do not. But it seems to us that such a distinction is questionable. It could be argued that contradictory statements are part of the world in the same sense as rocks, mountains and tables are. But we will not pursue this objection here. Instead, it will be convenient to take a better look at the details of axiomatic systems, in order to appreciate in what way they have been absorbed into the general notion of a syntactic system.

\section{Axiomatic Systems}

So what is an axiomatic system, exactly? According to Klimovksy and Boido, it has five components: a specific vocabulary, an underlying logic, well-formed formulas, axioms, and theorems.

10 Klimovsky and Boido, Las desventuras del conocimiento matemático, 122. We have translated this quote into English, as well as the rest of the quotes from their book.

11 Klimovsky and Boido, Las desventuras del conocimiento matemático. Filosofía de la matemática: una introducción, 123.

12 Meillassoux uses a French edition of Da Costa's work, Logiques classiques et non-classiques. Most of Da Costa's work is written in Portuguese, but there are some English translations, such as his article On the theory of inconsistent formal systems. In articles such as that one, he does not use the term "paraconsistent", but instead explores the formal aspects of inconsistent systems. The term "inconsistent", in that context, is not pejorative. 
The specific vocabulary of a formal axiomatic system is a set of meaningless signs, which is divided into two subsets: the subset of primitive terms, and the subset of defined terms. Klimovsky and Boido state that in Hilbert's formalization of Euclidean geometry, the terms "point", "line", "plane" and such are primitive terms. Contrary to Euclid's own geometry, in Hilbert's reformulation these terms do not mean anything. They could be replaced by the terms "beer", "table", and "salt", and this re-naming would not alter the operations we can make with them. Thus, a primitive term is an undefined term, it is a meaningless term in the sense that it does not have a reference, which is to say, it has no semantic aspect. Primitive terms do not denote.

The other subset of the specific vocabulary is the subset of defined terms. Here the term "defined" can be misleading, because defined terms are just as meaningless as primitive terms, which is to say, they do not denote either. They have no reference. We call them "defined" only in the sense that they are derived from the primitive terms of the axiomatic system. The example that Klimovksy and Boido mention is once again taken from Hilbert's axiomatization of Euclidean geometry. The term "plane", they say, is a primitive term, but the term "circumference on a plane" is a defined term, precisely because it is defined by the primitive terms of the system, terms such as "plane". So, both the primitive terms and the defined terms are meaningless.

The underlying logic provides an additional subset of meaningless terms. If, for example, we are using propositional logic, then the subset of logical terms includes the following meaningless signs: p, q, r, -, (, ), $\rightarrow$, v, among others. The underlying logic also specifies which combinations of meaningless signs are wellformed formulas of the axiomatic system. Finally, it also states which are the rules of deduction, or rules of inference. In other words, it specifies the valid forms of arguments. For example, modus ponens and modus tollens are valid arguments or rules of deduction in propositional logic, and in other logics as well, such as predicate logic.

The well-formed formulas of an axiomatic system are combinations of meaningless signs that follow the rules of the underlying logic. If we are using propositional logic, then a well-formed formula is this one: " $p \rightarrow q$ ". A formula that is not well formed is this one: " $\rightarrow \mathrm{p} q$ ". If we are using a natural language, such as English, an example of a well-formed formula is this: "from a point exterior to a line, there can be one and only one line which is parallel". An example of a formula that is not well-formed is this: "point line exterior parallel a from to".

The axioms are a special group of well-formed formulas, or, more precisely, they are a subset of well-formed formulas which are used as the starting points of the axiomatic system. As an example, Klimovksy and Boido invent a simple formal axiomatic system, which they call $\mathrm{SAFO}^{13}$, in order to highlight the different components that compose such systems in general. Their system has the following two axioms:

1. $(\forall \mathrm{x})-\mathrm{xRx}$

2. $(\forall \mathrm{x})(\forall \mathrm{y})(\forall \mathrm{z})[(\mathrm{xRy}) \wedge(\mathrm{yRz})] \supset \mathrm{xRz}$

The first axiom characterizes the property of anti-reflexivity. That is, it states that no individual is related to itself by the relation " $R$ ". That relation can have many different interpretations. For example, if " $R$ " is interpreted as "the father of", then it is true that no individual is his own father. Or, to use a numerical example, if "R" is interpreted as ">" (greater-than), then it is true that no number is greater-than itself. The second axiom characterizes the property of transitivity. It says that if " $x$ " has a relation " $R$ " with " $y$ ", and if " $y$ " has the same relation " $R$ " with " $z$ ", then " $x$ " has the relation "R" with "z". Again, there are many possible interpretations of this axiom. If " $R$ " is interpreted as "taller than", then it is true that if " $x$ " is taller than " $y$ ", and if " $y$ " is taller than " $z$ ", then " $x$ " is taller than " $z$ ". To give a numerical example: if " $\mathrm{R}$ " is interpreted as "greater-than", then it is true that if $x>y$, and if $y>z$, then $x>z$.

13 An acronym for "sistema axiomático formal para el orden" (formal axiomatic system for order). It should be noted that, while Klimovsky and Boido do not explicitly say so, this acronym coincides with the name of the Greek lesbian poet Sappho, whose name in Spanish is usually written as "Safo". Even though they do not explicitly mention this coincidence, it seems like it was a deliberate tribute to her nonetheless. 
Finally, the theorems are well-formed formulas that are deduced from the axioms of the system, using the underlying logic in order to perform those deductions. In the example provided by Klimovsky and Boido, we can deduce the following theorem:

Theorem (from axioms 1 and 2): $(\forall x)(\forall y) x R y \supset-y R x$

This theorem characterizes the property of asymmetry. It says that if $\mathrm{x}$ has the relation " $\mathrm{R}$ " with respect to " $y$ ", then " $y$ " does not have the relation " $R$ " with respect to " $x$ ". For example, if " $R$ " is interpreted as "the father of", then it is true that if " $x$ " is the father of " $y$ ", " $y$ " is not the father of " $x$ ". Another example: if " $R$ " is interpreted as "greater-than", then it is true that if $\mathrm{x}>\mathrm{y}$, then it is not the case that $\mathrm{y}>\mathrm{x}$.

According to Klimovsky and Boido, the main consequence that formal axiomatic systems have for philosophy is that the Aristotelian notion of truth must be abandoned. More precisely, Aristotle thought that axioms are self-evident, true statements, and that theorems are true statements that are derived from the axioms. In formal axiomatic systems, one can no longer say that an axiom or a theorem is "true" in the Aristotelian sense. Axioms and theorems are meaningless well-formed formulas, chosen arbitrarily. In the preceding example of the SAFO system, there is no reason to formulate the properties of anti-reflexivity and transitivity as axioms. One could invent a different system in which reflexivity and symmetry are axioms instead, for example, or one in which asymmetry is an axiom instead of a theorem. The point is that one can arbitrarily start from any axioms one wishes. Now we can take a better look at syntactic systems.

\section{Syntactic Systems}

Recall that formal axiomatic systems are a subset of syntactic systems, and that the difference between them is that the former have an underlying deductive logic, while the latter have production rules. The latter, as Klimovsky and Boido suggest, "are not necessarily guided by grammatical or logical considerations". So, for example, we could invent a syntactic system in which we use these three meaningless signs: *, @, \#. We could then propose a production rule which says that two clovers is a well-formed formula, and we could even say that this meaningless expression is an axiom of our syntactic system. Even more so, we could say that from ** and \#\# we may obtain @\# as a result. What grammatical or logical considerations have guided these decisions? None whatsoever. These decisions were purely arbitrary.

In propositional logic, symbols such as “p”, q”, “-”, “-”, among others, are meaningless signs, just as “*”, “@”, and “\#” are. And the combinations of those signs are just as conventional and arbitrary as those of the latter. A well-formed formula such as " $p \rightarrow(q \rightarrow r)$ " is just as conventional and arbitrary as "*s*". Even more so, one could use the symbols of propositional logic in order to build a non-classical logic, where a series of meaningless signs such as " $(\rightarrow$ ( p q r" would be a well-formed formula. Such an expression is not a well-formed formula in propositional logic, but it could be one in a new, non-classical logic.

We will show that the concept of a syntactic system provides some interesting tools for understanding Meillassoux's concept of hyper-Chaos. In order to do this, we must first start by actually inventing a syntactic system. Ours will be a very simple one, which nonetheless will suffice for our purpose. First, we arbitrarily choose three meaningless signs for our syntactic system. We have already used them in the previous paragraphs:

1. Meaningless signs: @, \#, *

Now we arbitrarily decide which combinations of these signs will be well-formed formulas of our system. We simply choose to establish a minimum of one and a maximum of two signs for all of the expressions of the system: 
2. Meaningless well-formed formulas: @, \#, *, @@, @\#, @*, \#\#, \#@, \#*, **, *@, *\#, @, \#, *.

Those are the only well-formed formulas of our syntactic system. A formula which is not well formed is, for example, @*@. Why? We have chosen as well-formed formulas any single meaningless sign and also any pair of meaningless signs, so that any expressions that contain more than two signs will not count as well-formed formulas in our system. Is there a reason for this? No, there is not. It is purely arbitrary and conventional. We could have chosen instead to define the well formed-formulas as expressions containing three signs. Or we could have chosen only some pairs of meaningless signs to be well-formed formulas, but not others. Note that in our system, a single meaningless sign also counts as a well-formed formula.

From the preceding formulas, we select only a few of them as axioms, that is, as starting points. The axioms that we arbitrarily choose are the following ones:

\section{Axioms: @@,\#\#, ***}

Now we define the production rules. All of them will be a combination of two axioms, and each combination will produce a different theorem. We will only give two examples:

4. Production rules

I)@@

II) \#\#

Therefore, III) *\#

I) $\% *$

II) \#\#

Therefore, III) \#@

Finally, we have the theorems. We have already stated them, but we may choose to highlight them, give them a name, and indicate how we obtained them:

\section{Theorems}

Clover-Hashtag theorem: *\# (obtained from the double-at and double-hashtag axioms)

Hashtag-At theorem: \#@ (obtained from the double-clover and double-hashtag axioms)

If at this point the reader asks "What does the clover mean in the preceding system?", the answer is that it does not mean anything: it is a meaningless sign. Why were these signs chosen instead of others? For no logical reason whatsoever. Maybe we had certain aesthetic or psychological motives for doing so. But from a purely formal point of view, they were arbitrary choices, meaning that there is no logical "rule" which forces us to choose certain signs instead of others, or certain well-formed formulas instead of others. Even if we had aesthetic or psychological reasons for these choices, from a logical point of view any choice would have been equally legitimate.

Syntax, understood in the preceding sense, offers limitless creativity, because any sort of syntactic system can be invented at will. Having said that, there seem to be some constraints on syntactic systems, nonetheless. One of those constraints is the requirement that there be axioms; in other words, that we must select a subset of well-formed formulas as axioms. But is this necessary? Must syntactic systems have axioms? The answer is "no". Yet, if we do not have any axioms, from where do we start?

We do not need to chose some subset of the well-formed formulas as axioms. The rationale for this claim is provided by what has been called "natural deduction", which was developed in the first half of the 20th century. Natural deduction is the name of a proof calculus which was invented by Gerhard Gentzen. ${ }^{14}$ He was dissatisfied with Hilbert's and Russell's axiomatic approach to mathematics, and his

14 Gentzen, "Untersuchungen über das logische Schließen. I”. 
idea was to develop an alternative approach, one in which axioms were not used. Instead of axioms, we may rely solely on the production rules, by adding an additional one called "rule of assumptions". This rule allows us to use any well-formed formula as a starting point. ${ }^{15}$ As Susan Haack says in Philosophy of Logics:

An axiomatic system of logic (e.g. Principia Mathematica) includes, besides one or more rules of inference, a privileged set of wffs, the axioms, which may be used at any point in an argument, and the truth of which is unquestioned in the system. The axioms are included among the theorems of the system, since, trivially, they are derivable from themselves. (An axiomatic system must have at least one rule of inference, since no derivations or proofs would be possible without the means to move from one wff to another.) A natural deduction formulation (e.g. Beginning Logic), by contrast, relies just on rules of inference. (A rule of assumptions will enable one to get started without the need of axioms from which to begin.) ${ }^{16}$

Other authors, such as Paul Tomassi ${ }^{17}$, trace a distinction between the rule of premise-introduction and the rule of assumptions. The first of these enables one to get started by stating a premise, the second by stating an assumption. Briefly put, the difference would be that once the conclusion is reached, it should only depend on the premises, not on the assumptions. The details of this technical discussion, while important in themselves, are beyond the scope of this essay. Here we will simply use the term "rule of assumptions" without tracing a technical distinction between propositions and assumptions.

The rule of assumptions for our syntactic system would be this: "Any meaningless sign or well-formed formula of the system can be postulated as a starting point". This being so, we have eliminated the axioms from the components of our syntactic system. Can we eliminate more components in order to arrive at something even more abstract?

The answer is yes. In our example, we may replace the production rules (except for the rule of assumptions) with this other rule: "Any meaningless sign or well-formed formula of the system can be produced from any other meaningless sign or well-formed formula of the system". Thus, we now have only two production rules; one of them is the rule of assumptions, and the other is the one we have just formulated. These two rules imply that, whatever starting point we choose, we can obtain anything we want from it. So, for example:

I)@

Therefore, II) \#*

We chose “@” as a starting point, and we obtained “\#*” from it. We could have obtained, instead, “\#@”, or "*", or "*w", or any other meaningless sign or well-formed formula of the system.

What is the point of this? Why would we want to obtain any well-formed formula from any other well-formed formula whatsoever? We have two reasons for doing this. One of them is to show that the arbitrariness and abstraction that according to Klimovsky and Boido characterizes a syntactic system can be increased. The other one is to show that this procedure will enable us to develop a purely syntactic version of Meillassoux's concept of hyper-Chaos.

\section{Formal Hyper-Chaos and Interpreted Hyper-Chaos}

In After Finitude, the concept of hyper-Chaos has a semantic aspect; it is already something interpreted, because it refers to entities, things, processes, situations, etc. The syntactic version that we will develop, in contrast, does not refer to anything. It has no interpretation. We arrive at it by a procedure of increasing

15 Let us note in passing that natural deduction could have profound consequences for Badiou's philosophy, given the importance that he gives to axioms, specifically to axiomatic set theory. See his Being and Event. We leave open as a question for the reader what would happen to Badiou's philosophy if his axiomatic approach were to be replaced by natural deduction, in which one does not need to accept any axioms whatsoever.

16 Haack, Philosophy of Logics, 19. The term "wffs" is an acronym for "well-formed formulas”.

17 Tomassi, Logic, 59. 
abstraction and arbitrariness, and the starting point for that is the concept of a syntactic system. The procedure itself consists in a step by step elimination of its components.

First we eliminated the axioms. However, we had also seen that a syntactic system has production rules that indicate which meaningless expressions may be produced, and how. No matter how arbitrary those rules may be, they are rules nonetheless. We simplified those rules, replacing them with the rule of assumptions and the rule that allows us to obtain any well-formed formula from any other well-formed formula. This would be akin to the Meillassouxian thesis that an entity can radically become anything else for no reason whatsoever, like a billiard ball that suddenly turns into a swan, for example.

So let us continue with our procedure. Now it is time to take out another component of the system, the well-formed formulas. We now state the following: "Any combination of the meaningless signs of the system is a well formed-formula". What this means is that, using the meaningless signs of the system, the distinction between a well-formed formula and a non-well-formed formula is blurred. Expressions such as “@\#*”, “@@@*@@@*”, and “\#*\#*\#** are now permitted. So, for example:

I) @\#@\#@\#

Therefore, II)*@\#*@*@\#

At this point, we can see where this is going. Now we can obtain whatever we want from whatever we postulate, so long as the meaningless signs of the system are used. But we can take things further by eliminating what we consider to be the meaningless signs of the system, by stating the following: "any visual mark is a meaningless sign of the system". This includes letters, numbers, and any other mark. So, for example:

I) $/ 63 \% \& " 824 \mathrm{r}$

II) $(737621 \& \% \$ \$(/$

III) HFJKRbha87436538hauwDXFBb

We have arrived at high degree of arbitrariness, and we are no longer in the presence of a syntactic system. What we have before us is the possibility of making any visuals marks that we want, and of obtaining any other visual marks that we want from them. This could lead to entire pages of meaningless signs, a completely arbitrary and chaotic text. But since any sequence of visual marks is permitted, this also allows us to introduce visual marks in such a way that they resemble intelligible and readable text. So, for example:

I) It was a quiet day at the office.

II) Henry ate an apple.

We can also alter the ordering of the preceding visual marks in any way that we want. So, for example:

I) Day it the quiet was office a.

II) Apple ate Henry.

Even more bizarrely, we could write:

I) 8762 \#”(/\& Office, Day “/”\$ Henry

II) Apple*@\#*@ate (\&”

We are now very far from the notion of a syntactic system as Klimovsky and Boido characterize it. Of course, we are also very far from a system of natural deduction. What is this, then? Let us call it "formal hyperChaos”. It has the four following characteristics:

1. Meaningless signs: Any visual mark is a meaningless sign of the system.

2. Meaningless expressions: Any combination of visual marks is a meaningless expression of the system.

3. Production rule: Any meaningless expression of the system can be obtained from any other meaningless expression of the system.

4. Rule of assumptions: any meaningless sign or meaningless expression can be postulated as a starting point. 
When one thinks of chaos, this notion generally connotes disorder and randomness. However, Meillassoux says that this is only one aspect of hyper-Chaos. It certainly may produce disorder and randomness, but not necessarily. It can also produce an orderly universe. If we were to use the concept of formal hyperChaos strictly for literary purposes, we could say that it would not be necessary for hyper-chaotic texts to be entire pages of random successions of meaningless signs. It certainly could be that, and there would not be anything wrong with it. But it would not necessarily have to be that and only that. On the contrary, a strictly realist novel, in the sense of 19th century literary realism, would also count as hyper-Chaotic literature.

On the other hand, we can get back to the clover-at-hashtag system by following an inverse procedure; that is, by introducing a series of restrictions, step by step. What we initially eliminated from the system can be put back into it. So, if we start from the concept of formal hyper-Chaos, we can introduce this first restriction: “The meaningless signs of the system are @, \#, *.” In other words, it is no longer legitimate to claim "any visual mark is a meaningless sign of the system". After incorporating these meaningless signs, the next step is to introduce the well-formed formulas. So expressions that have more than two meaningless signs will now not be permitted. We then incorporate the production rules that we had previously eliminated. And finally, we may choose to replace the rule of assumptions by the axioms.

Analogous restrictions can be incorporated into the concept of formal hyper-Chaos in order to arrive at the two large branches that are logic and mathematics. Meillassoux himself introduces restrictions into his version of hyper-Chaos; for example, that a contradictory entity cannot be produced. But instead of reconstructing his concept of hyper-Chaos step by step using those restrictions, we will now turn to a different issue, that of ontology. One of the most famous aspects of Hilbert's mathematical formalism is that it has no ontology whatsoever: numbers in particular, and meaningless signs in general, do not refer to anything. As long as pure mathematics has no interpretation, its constitutive elements have nothing to do with the existence of mathematical entities, either in a Platonic "realm of Ideas" or as incorporeal, ghostly quantities attached to things in the real world. However, Meillassoux claims that mathematical formalism does indeed have an ontology, albeit a rather strange one: it is an ontology of meaningless signs. This was the topic of his Berlin lecture of 2012. But we will not delve into this here.

Formal systems, such as non-Euclidean geometries, can have interpretations. One could, in principle, invent a syntactic system and then proceed to give that system a physical interpretation. The crucial question, however, is if the concept of formal hyper-Chaos can have a physical interpretation. To us, at least, it seems that this is what Meillassoux is actually claiming, even if he does not phrase it in this way. Keeping in mind the characteristics of formal hyper-Chaos, we can give them the following one-to-one interpretation:

1. Any entity, relation, process, fact, situation, etc., is a meaningless component of the Universe.

2. Any combination of meaningless components of the Universe is a meaningless composite of the Universe. Thus, things can radically combine themselves with other completely different things, for no reason whatsoever.

3. Any meaningless composite of the Universe can produce or be transformed into any other meaningless composite of the Universe. Thus, things can radically change for no reason whatsoever.

4. Any meaningless component or meaningless composite can appear as a starting point. Thus, emergence from nothing is possible.

The arbitrariness and randomness that characterizes formal hyper-Chaos is now interpreted in order to describe a Universe which is just as arbitrary and random. But, just like the most ordered texts such as realist novels and scientific papers qualify as instances of hyper-Chaotic texts, the order and stability that we see in the Universe is also under the scope of the interpreted hyper-Chaos. To us, this seems like the most precise way in which Meillassoux's concept of absolute contingency, poetically called "hyper-Chaos", can be understood: as the semantic interpretation of a purely formal hyper-Chaos. 


\section{Concluding Remarks}

Whether or not such an interpreted hyper-Chaos actually exists is another matter, and only the sciences, such as physics and astronomy, can determine this. We are not claiming that interpreted hyper-Chaos actually exists. We are merely saying that Meillassoux's version of that concept can be divided into two separate facets: a purely syntactic one and a semantic one. It is not our task to determine if the semantic version of hyper-Chaos actually refers to something real, although we highly doubt that this is the case. But this, by itself, cannot be a reason for not examining Meillassoux's philosophy with precision and careful scrutiny.

\section{Reply to a Possible Objection}

One of the reviewers of our manuscript has formulated a possible objection, which we have to address. In their own words:

I think that from Meillassoux's point of view there is a specific reason why we cannot divide his hyper-chaos in two separate facets, a purely formal one and a semantic (ontologically interpreted) one. The reason is that the concept of 'hyperchaos' operates at the transcendental, not the empirical level. In Heideggerian terminology, it is an 'ontological', not 'ontic' truth. Thus, e.g. the author's view to the effect that "whether or not such (i.e. Meillassouxian) interpreted hyper-chaos actually exists is another matter, and only the sciences such as physics or astronomy can determine this" (p. 12), is not just false but impossible from Meillassoux's standpoint. From this latter point of view thus there is a deeper structural reason why a) we cannot divide 'hyper-chaos' in two separate levels, a formal and a semantic one, and b) a fortiori, we cannot privilege the formal level as less 'speculative' or less ontologically committal than the semantic one. ${ }^{18}$

Let us begin by addressing the difference between the transcendental and the empirical. Meillassoux himself has discussed this issue in relation to a possible objection against his own line of reasoning:

We shall formulate the second correlationist rejoinder from a transcendental perspective, which here constitutes the more incisive objection to our argumentation:

'Your objection, made in the name of the arche-fossil, evinces an elementary confusion between the empirical and the transcendental level of the problem under consideration.

'The empirical question is that of knowing how bodies that were organic prior to becoming conscious appeared in an environment which is itself physical. The transcendental question consists in determining how the science of this physical emergence of life and consciousness is possible. Now, these two levels of thought - the empirical and the transcendental are like the two faces of a flat sheet of paper: they are absolutely inseparable but they never intersect. But your mistake consists precisely in allowing them to intersect - you have turned a structure which should have remained flat into a Möbius strip. You proceed as though the transcendental subject - which is ultimately the subject of science - was of the same nature as the physical organ which supports it - you collapse the distinction between the conscious organ which arose within nature and the subject of science which constructs the knowledge of nature. But the difference between these two is that the conscious organ exists; it is an entity in the same sense as any other physical organ; whereas the transcendental subject simply cannot be said to exist; which is to say that the subject is not an entity, but rather a set of conditions rendering objective scientific knowledge of entities possible. But a condition for objective cognition cannot be treated as an object, and since only objects can be said to exist, it is necessary to insist that a condition does not exist - precisely because it conditions. ${ }^{19}$

The objection continues for another page, with more details and nuances, but the general idea has been spelled out: the transcendental and the empirical are to be kept neatly separated. Meillassoux provides an answer to that objection:

The subject is transcendental only insofar as it is positioned in the world, of which it can only ever discover a finite aspect, and which it can never recollect in its totality. But if the transcendental subject is localized among the finite objects of its

18 Reviewer, personal communication.

19 Meillassoux, After Finitude, 22-23. 
world in this way, this means that it remains indissociable from its incarnation in a body; in other words, it is indissociable from a determinate object in the world. Granted, the transcendental is the condition for knowledge of bodies, but it is necessary to add that the body is also the condition for the taking place of the transcendental. That the transcendental subject has this or that body is an empirical matter, but that it has a body is a non-empirical condition of its taking place the body, one could say, is the 'retro-transcendental' condition for the subject of knowledge. We will invoke an established distinction here and say that a subject is instantiated rather than exemplified by a thinking body. ${ }^{20}$

For our part, and in response to the reviewer, we may say that Meillassoux's philosophy is quite in favor of turning flat structures into Möbius strips, as long as it is shown that the transcendental is indissociable from an object in the world. Of course, he is discussing the specific case of the transcendental subject and its body, but his remarks can be extended in a way that encompasses the concept of hyper-Chaos. Even if his version operates at the transcendental level, as the reviewer argues, this does not mean that it can be dissociated from an object in the world.

Speaking of the transcendental subject, it does not matter if it has this or that body, since, as Meillassoux says, this is an empirical issue. What is not empirical is that it has some body, whichever it may be. Similarly, it does not matter which object in the world is indissociable from hyper-Chaos, since that would also be an empirical issue. But similarly again, that it is indissociable from some object, whichever it may be, is not empirical. And it just so happens that according to Meillassoux, every object in the world is indissociable from hyper-Chaos, since he claims that the latter is the sole, absolute and eternal property of every entity. Hyper-Chaos is not exemplified in particular objects, it is instantiated in them, and this means that it cannot exist independently of them. And it is precisely because of this that he is able to provide the following answer to the question of why there is something rather than nothing:

Thus the solution to the problem is as follows: it is necessary that there be something rather than nothing because it is necessarily contingent that there is something rather than something else. The necessity of the contingency of the entity imposes the necessary existence of the contingent entity. ${ }^{21}$

The reviewer suggests that Meillassoux’s hyper-Chaos is, using Heideggerian terminology, an "ontological" truth, not an "ontic" one. We have no quarrel with this. The question that should be formulated at this point is: how does Meillassoux conceive the truth of the ancestral statements that are formulated by the empirical sciences? Are these truths, according to him, ontological or ontic? There is no explicit answer to this question in Meillassoux's works. He does, however, address this conceptual difference in relation to mathematics:

Consequently, we are faced with the requirement of a twofold absolutization of mathematics. The absolutization inherent in the problem of diachronicity basically asserts that every mathematical statement describes an entity which is essentially contingent, yet capable of existing in a world devoid of humanity - regardless of whether this entity is a world, a law, or an object. Accordingly, this is an absolutization that could be called ontical: it pertains to entities that are possible or contingent, but whose existence can be thought as indifferent to thought. By way of contrast, the absolutization of the Cantorian non-All requires an absolutization that is ontological, rather than ontical, because it now states something about the structure of the possible as such, rather than about this or that possible reality. ${ }^{22}$

At the risk of distorting Meillassoux's phrasing, we can say that he believes that there are two kinds of mathematical statements: those that are true in an ontic sense, and those that are true in an ontological sense. If we must utilize Heideggerian terminology, then we may say that our concept of formal hyper-Chaos is composed of statements that are true in the ontological sense, while interpreted hyper-Chaos is composed of statements that are true in an ontic sense, but only if they refer to something that actually exists. This last issue is not ontological, it is ontical. And since the empirical sciences deal with ontic truths, this is what enables them to determine if interpreted hyper-Chaos actually exists, in other words if the statements

20 Ibid., 25.

21 Ibid., 76.

22 Ibid., 127. 
in question have a reference. The reviewer is therefore wrong in believing that the preceding claim is false and impossible from Meillassoux's standpoint. Quite the contrary, it is entirely compatible with it. In other words, they have mistakenly assumed that we were implying that interpreted hyper-Chaos is ontological, when it is the other way around: it is ontical.

We fully agree with the reviewer when they say that "we cannot privilege the formal level as less 'speculative' or less ontologically committal than the semantic one." But they are wrong in believing that we ever denied this in the first place. What we did say was that mathematical formalism, specifically in the style of Hilbert and his successors, claims that mathematics has no ontology whatsoever. And we also said that Meillassoux challenged this point of view in his Berlin lecture, where he said that formalism entails an ontology of meaningless signs. As for our own point of view on this issue, we have remained entirely silent. But if we must disclose our personal opinion, we may say the following: we believe that Meillassoux is on the right track in his critique of mathematical formalism, even if he has taken some wrong steps. There does seem to be an ontology to formalism, not only mathematical, but also logical ("syntactical", if you will). But Meillassoux's argumentation hinges on the idea of "perceiving contingency", not by the senses, but by the mind. In After Finitude, he had already advanced this idea of "intellectual intuition", which, ever since Kant, has become highly dubious: nothing seems to indicate that human beings have such a faculty.

Formal hyper-Chaos no doubt will show its Achilles's heel sooner or later, and we do not need science to uncover its shortcomings; philosophy suffices for this purpose. But the existence of interpreted hyperChaos, being on the ontic rather than ontological side of this Heideggerian spectrum, is something that should be determined by disciplines that deal with ontic truths, the empirical sciences.

\section{References}

Aristotle. Aristotle's Posterior Analytics. Translated by E. S. Bouchier, B. A. Oxford: Blackwell, 1901.

Badiou, Alain. Being and Event. Translated by Oliver Feltham. Continuum: London, 2005.

Carnap, Rudolf. Logical Syntax of Language. Reprinted. Translated by Amethe Smeaton. Routledge: London, 2001.

Carnap, Rudolf. “Intellectual Autobiography”. In Schilpp, Paul Arthur, ed. The Philosophy of Rudolf Carnap. Third Printing. La Salle, Illinois: Open Court, 1997, 1-84.

Da Costa, Newton Carneiro Affonso. "On the theory of inconsistent formal systems". Notre dame journal of formal logic, 1974 15(4): 497-510.

Da Costa, Newton Carneiro Affonso. Logiques classiques et non-classiques. Paris: Masson, 1997.

Deleuze, Gilles; and Guattari, Félix. What is Philosophy? Translated by Hugh Tomlinson and Graham Burchell. Columbia University Press: New York, 1994.

Gentzen, Gerhard Karl Erich. “Untersuchungen über das logische Schließen. I”. Mathematische Zeitschrift. 193439 (2): 176-210.

Haack, Susan. Philosophy of Logics. Cambridge University Press: Cambridge, 1978.

Heidegger, Martin. Being and Time. Reprinted. Translated by John Macquarrie \& Edward Robinson. Blackwell: Oxford UK, 2001.

Klimovsky, Gregorio, \& Boido, Guillermo. Las desventuras del conocimiento matemático. Filosofía de la matemática: una introducción. AZ Editora: Buenos Aires, 2005.

Meillassoux, Quentin. After Finitude. An Essay on the Necessity of Contingency. Translated by Ray Brassier. Continuum: London, 2008.

Reisch, George. How the Cold War Transformed Philosophy of Science. To the Icy Slopes of Logic. Cambridge University Press: New York, 2005.

Tomassi, Paul. Logic. Published in the Taylor \& Francis e-Library. Routledge: London and New York, 2002. 